\title{
Oscillating grids turbulence generator for turbulent transport studies
}

\author{
A. Eidelman, T. Elperin, A. Kapusta, N. Kleeorin, A. Krein, and I. Rogachevskii \\ The Pearslstone Center for Aeronautical Engineering Studies, Department of Mechanical Engineering, Ben Gurion University \\ of the Negev, P. O. Box 653, Beer-Sheva 84105, Israel
}

Received: 12 September 2001 - Revised: 12 November 2001 - Accepted: 14 November 2001

\begin{abstract}
An oscillating grids turbulence generator was constructed for studies of two new effects associated with turbulent transport of particles, turbulent thermal diffusion and clustering instability. These effects result in formation of large-scale and small-scale inhomogeneities in the spatial distribution of particles. The advantage of this experimental set-up is the feasibility to study turbulent transport in mixtures with controllable composition and unlimited observation time. For flow measurements we used Particle Image Velocimetry with the adaptive multi-pass algorithm to determine a turbulent velocity field and its statistical characteristics. Instantaneous velocity vector maps, flow streamlines and probability density function of velocity field demonstrate properties of turbulence generated in the device.
\end{abstract}

\section{Introduction}

In our previous studies (Elperin et al., 1996a, 1996b, 1998a, $1998 \mathrm{~b}, 2000 \mathrm{a}, \mathrm{b})$ we predicted a number of new effects associated with turbulent transport of particles. These effects, namely turbulent thermal diffusion and clustering instability, are related with the interaction between inertial solid particles and turbulent flows and result in formation of large-scale and small-scale inhomogeneities in particles spatial distribution.

Turbulent thermal diffusion is caused by the correlation between temperature and velocity fluctuations in the surrounding fluid and leads to a relatively strong non-diffusive mean flux of inertial particles in regions with mean temperature gradients. Particles are accumulated in the vicinity of a minimum of the mean temperature of the surrounding fluid, which results in the formation of large-scale inhomogeneities.

Clustering instability is caused by a combined effect of the particles inertia and a finite correlation time of the velocity field (compressibility of Lagrangian trajectories). It

Correspondence to: A. Eidelman (eidel@bgumail.bgu.ac.il) results in the growth of the number of particles in the smallscale clusters. This growth is saturated by particle collisions and other non-linear mechanisms. Small-scale (of the order of several Kolmogorov scales) inhomogeneities in the spatial distribution of the particles can be important in various atmospheric phenomena, such as rain formation or scavenging of pollutants. Notably, small-scale inhomogeneities of droplets distribution were observed in clouds (Baker and Brenguire, 1998; Kostinski and Shaw, 2001) and laboratory turbulent flows (Fessler et al., 1994; Hainaux et al., 2000).

The main goal of this communication is to describe an experimental set-up constructed for the verification of the effects of formation of large-scale and small-scale patterns in particle distribution and to present preliminary results of turbulence measurements in the device.

\section{Experimental set-up}

There exist two traditional methods for obtaining approximately homogeneous and isotropic turbulent flow in laboratory conditions. The first one uses grid generated stationary turbulence in a tunnel flow. However, the possible observation time in a wind tunnel is small and is determined by the flow passage time through the tunnel test section. Moreover, homogeneity is broken by the decay of turbulence downstream of the grid, and isotropy is violated by the directional flow in the wind tunnel.

Since our estimations showed that a characteristic time of observation of large-scale structures in spatial distribution of aerosol and gaseous must be relatively large, we employed oscillating grids to generate turbulence. Observation time in the oscillating grid turbulence generator is not limited due to a zero mean velocity of the flow.

Studies of turbulence generated by one oscillating grid in a water tank (see, e.g. Thompson and Turner, 1975) allowed to estimate the decay law of turbulence and dependence of turbulence parameters on the mesh size, frequency and amplitude of the grid oscillations. In particular, the linear depen- 


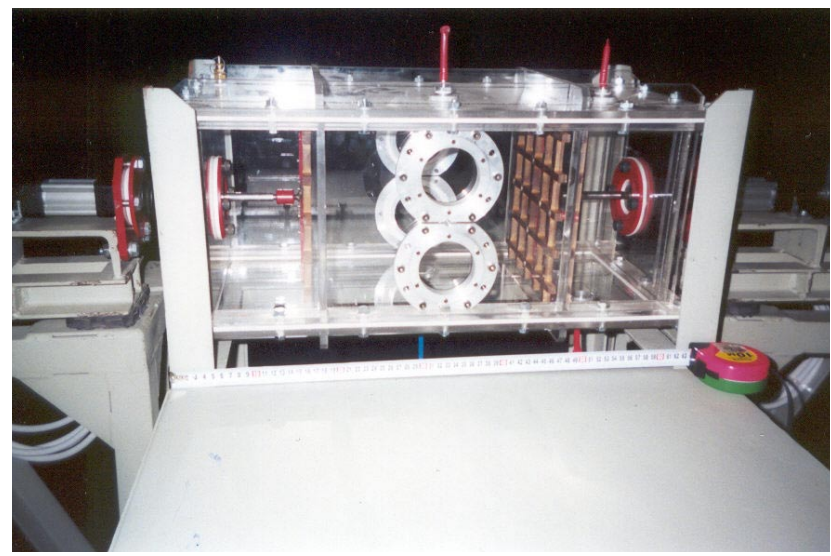

Fig. 1. Photograph of the oscillating grids turbulence generator.

dence of the r.m.s. turbulent velocity on the grid oscillations frequency was revealed by Thompson and Turner (1975) in the case of a square-bar grid.

It was found that the turbulent kinetic energy decays with distance from the grid according to a power law with the exponent -2 (Hopfinger and Toly, 1976). Turbulence intensity $u$ depends on the experimental parameters and the distance from a grid as follows:

$u=c_{u} c_{S}^{1.5} f M^{2} / z$,

where $c_{u} \approx 0.3, f$ is oscillations frequency, $S$ is the stroke, $M$ is the mesh size of the grid, $z$ is the distance from the grid mid-position, $c_{S}=S / M$. The turbulence integral scale, $l$, is proportional to the distance from a grid:

$l=c_{l} z$,

where $c_{l} \approx 0.12$. Recent studies of oscillating grid turbulence confirmed Relations (1) and (2) (see Kit et al., 1997). Studies of the oscillating grid turbulence revealed that it has the local properties of isotropic turbulence (De Silva and Fernando, 1994). A region of approximately homogeneous and isotropic turbulence is formed between two oscillating grids (Srdic et al., 1996). It was found (Shy et al., 1997) that the length of this region is linearly proportional to the distance between the grids.

Our estimations of the turbulence integral scale dependence based on data by Hopfinger and Toly (1976) vs. the distance $z$ in a range $S / M=0.2-0.9$ yield

$l=c_{o} c_{S}^{0.5} z$,

where $c_{0}=0.25$. Therefore, the Reynolds number, $R e=$ $u l / v$, using Eqs. (1) and (3) for the velocity and length scales, respectively,

$R e=\left(c_{u} c_{o} c_{S}^{2} / \nu\right) f M^{2}$,

where $v$ is the kinematic viscosity, does not depend on the distance from the grid. If $c_{u}=0.3, c_{o}=0.25, c_{S}=1$, and $v=0.15 \mathrm{~cm}^{2} / \mathrm{s}$, then $R e=0.5 f M^{2}$. The Relationships (1),
(3), and (4) for turbulence characteristics were used in the design of the experimental set-up.

Our experiments were carried out in a specially constructed set-up with oscillating grids turbulence generation (OGTG) in airflow. To the best of our knowledge this is the first OGTG constructed for conducting experiments in the gaseous flow. The advantage of this experimental set-up is the feasibility to study turbulent transport in gaseous mixtures with controllable composition at different temperatures and pressures and zero mean velocity.

A photograph of the OGTG is shown in Fig. 1, and other photographs can be found at http://www.bgu.ac.il/me/ laboratories/tmf/turbulentMultiphaseFlow.html. The OGTG comprises the following principal units: test-section, oscillated grids, grid drives and their control, devices for formation of the mean temperature gradient and for gaseous admixtures feeding, and instrumentation for control and measurements.

The test-section is constructed as a transparent rectangular chamber $75 \times 75 \times 150 \mathrm{~cm}$. A chamber with a smaller size $30 \times 30 \times 60 \mathrm{~cm}$ will also be used to test the sensitivity of the flow characteristics to the box size. Pairs of vertically oriented grids with bars arranged in a square array are attached to the right and left horizontal connecting rods supported individually by linear bearings. Both grids are driven independently or coupled at any constant phase shift with the speedcontrolled motors by means of eccentric cams supported with ball bearings. This two-grid system is capable of oscillating horizontally at controllable frequency up to $30 \mathrm{~Hz}$. The grid stroke is adjusted in a range up to $20 \mathrm{~cm}$. The maximum turbulence Reynolds number is of the order of 1500 .

Two heat exchangers for formation of the mean temperature gradient in the flow are placed on the bottom and top walls of the test section. They are connected to control devices in order to support a mean temperature gradient in the range up to $30 \mathrm{~K} / \mathrm{m}$ at a mean temperature of about $300 \mathrm{~K}$. Then, according to our theory, the gaseous admixture will accumulate at the region of the box with the minimum temperature. We designed and constructed a device for preparation and feeding of gaseous admixture into the turbulent flow. Preliminary experiments in non-isothermal gaseous mixture flow for validating the performance of the heat exchangers for producing constant mean temperature gradient and feeding device for forming initial homogeneous distribution of gaseous admixtures are under way.

The Richardson number of turbulence, $R i_{t}$, describes its evolution in a temperature-stratified flow

$R i_{t}=(N \tau)^{2}$,

where $\tau=l / u$ is the turbulence characteristic time, $N=\left(g \beta / T_{o}\right)^{1 / 2}$ is the Brunt-Vaisala frequency, $T_{o}$ is a characteristic temperature, $g$ the acceleration of gravity, and $\beta$ the temperature gradient. Relations (1) and (3) yield the following formula for the turbulence characteristic time

$\tau=0.8 f(z / M)^{2}$. 

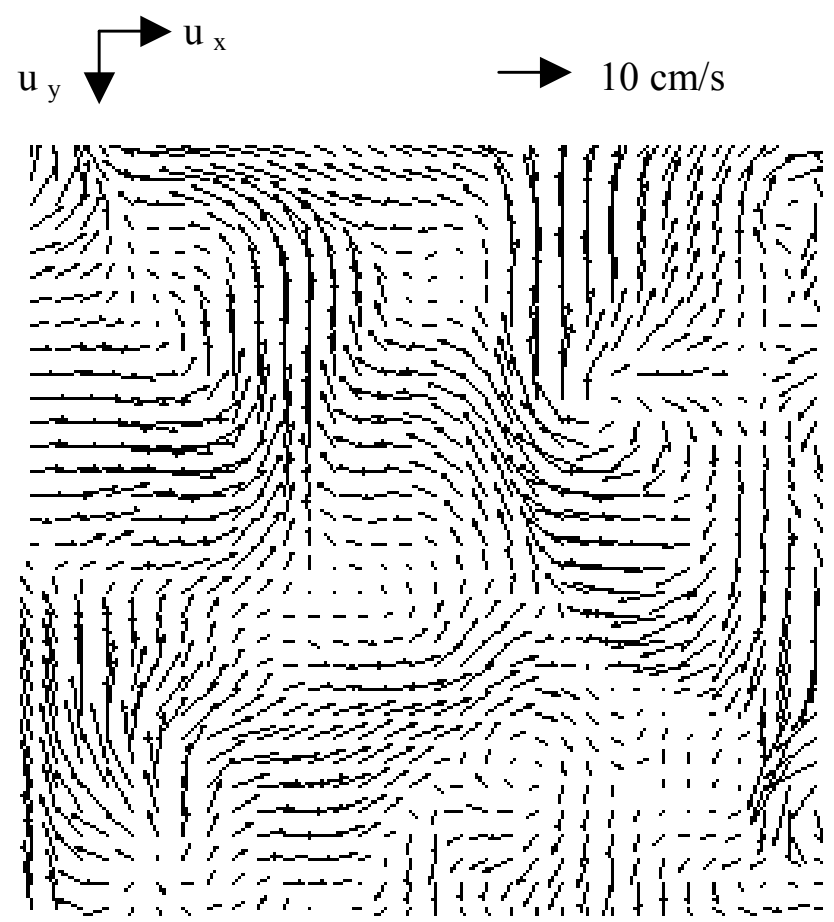

Fig. 2. The instantaneous velocity vector map shown with a grid of 32 pixels.

Relations (5) and (6) yield the following expression for the Richardson number at a distance $2 M$ from the grid:

$R i_{t}=10 N^{2} / f^{2}$.

If $R i_{t}<0.1$, then the flow is dynamically passive (Yoon and Warhaft, 1990). If the parameters of the experiment are $\beta=10 \mathrm{~K} / \mathrm{m}, g=9.8 \mathrm{~m} / \mathrm{s}^{2}, T_{o}=300 \mathrm{~K}$, then $N=0.57 \mathrm{~Hz}$. Therefore the flow is dynamically passive in this region at the grid oscillations frequency

$f=\left(10 / R i_{t}\right) 0.5 N>5 . \mathrm{Hz}$.

In the dynamically passive flow it is feasible to attain the temperature gradient $\beta$ up to

$\beta=0.1 T_{o} f^{2} / 10 g$,

e.g. $\beta=30 \mathrm{~K} / \mathrm{m}$ when $f=10 \mathrm{~Hz}$.

\section{Instrumentation}

For flow measurements we used Particle Image Velocimetry (PIV) (Raffel et al., 1998) to determine a turbulent velocity field and its statistical characteristics. These measurements will be repeated for various temperature gradients, Reynolds numbers, and gaseous admixture densities.

Thermoanemometers that are commonly used in wind tunnel experiments require a relatively large mean flow velocity in order to ensure the validity of Taylor's hypothesis. Hence, thermoanemometry can be used with limitation in the oscillating grid turbulence experiment. Laser Doppler velocimetry has the disadvantage that it allows only local measurements and does not allow to measure simultaneously velocity field in a region in a flow field.

PIV involves recording the Mie scattering from the seeding particles induced by two consecutive laser pulses. Small smoke particles with the size of the order of $1 \mu \mathrm{m}$, glass beads with a size of the order of $5 \mu \mathrm{m}$, and expanded particles with the size of order of $40 \mu \mathrm{m}$ were introduced as flow tracers into the air where turbulence was generated with the oscillating grids.

Turbulent velocity was measured using digital particle image velocimetry (DPIV) with LaVision system Flow Master III. A double-pulsed light sheet is provided by a $\mathrm{Nd}$ YAG laser source (Continuum Surelite $2 \times 170 \mathrm{~mJ}$ ). Light sheet optics comprises spherical and cylindrical Galilei telescopes with tuneable divergence and adjustable focus length. A progressive-scan $12 \mathrm{Bit}$ digital CCD camera $(1280 \times$ 1024 pixels of a size $6.7 \mu \mathrm{m} \times 6.7 \mu \mathrm{m}$ each) with dualframe-technique for cross correlation captures images. Programmable Timing Unit (a PC interface card) generates sequences of pulses to control the laser, the camera and data acquisition.

Software package DaVis 5 was applied to control all hardware components and for 32 bits image acquisition and visualization. DaVis 5 comprises PIV/PTV software for calculating the flow fields using a cross correlation analysis. Velocity maps and their characteristic statistics and PDF were analysed with this package.

We used the adaptive multi-pass algorithm that calculates first a reference vector field for each record to be processed. It starts with an initial interrogation window size, calculates the vectors there and uses the computed vector field information as the reference vector field for the next step. In the next pass the window size is half of the previous size. The vector calculated in the first pass is used as a best-choice cell shift for the second pass. This method for the interrogation window shift is adaptively improved to be able to compute the vectors in the following steps more accurately since less particles move inside or outside the window. Therefore, it is possible to use a much smaller final interrogation window size than would be possible without adaptive window shifting. This improves the spatial resolution of the vector field and produces less faulty vectors.

\section{Results}

Tracer velocities were measured in the flow generated by the oscillated grid in a smaller test section. One of the grids was removed and the light sheet ( $2 \mathrm{~mm}$ wide) was located in the $X Y$ plane parallel to the grid. Measurements in a flow generated by one grid are a necessary preliminary stage prior to measurements in a flow generated by two grids. Two components of velocity $u_{x}$ (horizontal) and $u_{y}$ (vertical) were measured in the light sheet plane (see Fig. 2). We intend to 


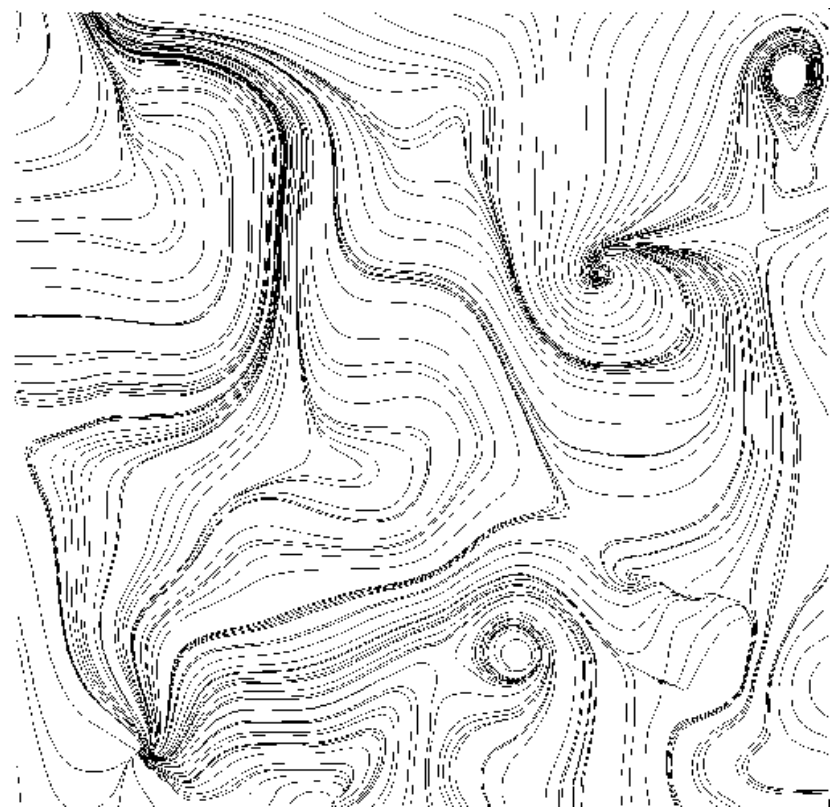

Fig. 3. Streamlines for the velocity map shown in Fig. 2.

measure also $u_{y}$ and $u_{z}$ velocity components in a light sheet plane $Y Z$ normal to the grid. We will use the same configuration of the light sheet and camera for turbulence measurements between two oscillating grids.

Turbulent flow velocity was measured at the distance $z=130 \mathrm{~mm}$ from the grid oscillating with frequencies $f=$ $4.45 \mathrm{~Hz}$ and $5 \mathrm{~Hz}$, and a stroke $S=10 \mathrm{~cm}$. An incense smoke with submicron particles was used for PIV measurements. The imaging areas were $70 \times 70 \mathrm{~mm}^{2}$ and $187 \times 187 \mathrm{~mm}^{2}$ corresponding to a linear magnification of 0.097 and 0.037 . The adaptive algorithm with the pass from the initial interrogation window with $32 \times 32$ pixels to $16 \times 16$ pixels was applied.

The instantaneous velocity vector map of the flow region $70 \times 70 \mathrm{~mm}$ at $f=4.45 \mathrm{~Hz}$ is showed in Fig. 2 with a grid of 32 pixels. Here large eddies with the size about $20 \mathrm{~mm}$ determining the integral scale of turbulence are clearly seen.

The streamlines of the same flow region shown in Fig. 3 demonstrate a wide range of scales of turbulent motions. Remarkably, the flow with wide range of spatial scales is formed already at the comparatively low frequency of grid oscillations. This shows that at higher frequencies it is feasible to obtain a fully developed turbulence in the oscillating grids generator in the air flow.

The PDF of the velocity component $u_{y}$ is shown in Fig. 4 . The PDF is Gaussian-like with several spikes. The spikes in the PDF are caused by peak locking that often occurs in PIV systems because the particle image diameter is smaller than the pixel size of the camera of CCD sensor array. We are currently improving the system in order to suppress this effect as much as possible.

There exists a weak mean flow in the $y$-direction. The mean flow can be seen in the $u_{y}$ velocity PDF shown in
254

number of vectors (total=4072)

0

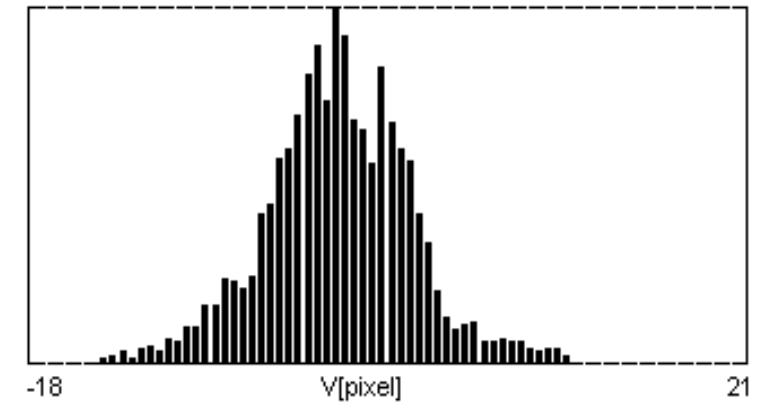

Fig. 4. Probability density function of $u_{y}$ for the velocity map shown in Fig. 2.

Fig. 4. It is very sensitive to the grid angle with the horizontal plane and the mean flow velocity can be controlled with the angle adjustment.

Measurements of velocity maps at different distances from the grid, for various values of strokes and frequencies are under progress. Series of 50-100 images are stored for calculating velocity maps and time averaging of turbulence characteristics. The results of our experiments showed that velocity components in the light sheet plane averaged over 50 images are fairly isotropic. Their r.m.s. value obtained at present in the limited range of distances is slightly less than expected from Relation (1).

Acknowledgements. We are indebted to E. Kit and A. Tzinober for illuminating discussions. This work was partially supported the German-Israeli Project Cooperation (DIP) administrated by the Federal Ministry for Education and Research (BMBF) and INTAS (Grant No. 00-0309).

\section{References}

Baker, B., Brenguier, J.-L., and Cooper, W.: Unknown source of reflectivity from small cumulus clouds, Proc. American Meteorology Society on Cloud Physics, Everett, Washington, 148-151, 1998.

De Silva, I. P. D. and Fernando, H. J. S.: Oscillating grids as a source of nearly isotropic turbulence, Phys. Fluids, 6, 24552464, 1994.

Elperin, T., Kleeorin, N., and Rogachevskii, I.: Turbulent Thermal Diffusion of Small Inertial Particles, Phys. Rev. Lett., 76, 224 227, 1996a.

Elperin, T., Kleeorin, N., and Rogachevskii, I.: Self-excitation of Fluctuations of Inertial Particles Concentration in Turbulent Fluid Flow, Phys. Rev. Lett., 77, 5373-5376, 1996 b.

Elperin, T., Kleeorin, N., and Rogachevskii, I.: Effect of Chemical Reactions and Phase Transitions on Turbulent Transport of Particles and Gases, Phys. Rev. Lett., 80, 69-72, 1998a.

Elperin, T., Kleeorin, N., and Rogachevskii, I.: Dynamics of Particles Advected by Fast Rotating Turbulent Fluid Flow: Fluctuations and Large-Scale Structures, Phys. Rev. Lett., 81, 28982901, 1998b.

Elperin, T., Kleeorin, N., Rogachevskii, I., and Sokoloff, D.: Passive Scalar Transport in a Random Flow with a Finite Re- 
newal Time: Mean-field Equations, Phys. Rev., E61, 2617-2625, 2000a.

Elperin, T., Kleeorin, N., Rogachevskii, I., and Sokoloff, D.: Turbulent Transport of Atmospheric Aerosols and Formation of LargeScale Structures, Physics and Chemistry of Earth A25, 797-803, 2000b.

Elperin, T., Kleeorin, N., Rogachevskii, I., and Sokoloff, D.: MeanField Theory for a Passive Scalar Advected by a Turbulent Velocity Field with a Random Renewal Time, Phys. Rev., E64, 026304(1-9), 2001.

Elperin, T., Kleeorin, N., L'vov, V., Rogachevskii, I., and Sokoloff, D.: Strong and Weak Clustering of Inertial Particles in Turbulent Flows, Europhysics Letters, submitted, 2002.

Fessler, J. R., Kulick, J. D., and Eaton, J. K.: Preferential Concentration of Heavy Particles in a Turbulent Channel Flow, Phys. Fluids 6, 3742-3749, 1994.

Hainaux, F., Aliseda, A., Carttellier, A., and Lasheras, J. C.: Settling velocity and clustering of particles in an homogeneous and isotropic turbulence, Advances in Turbulence VIII, Proceedings of the 8th European Turbulence Conference, Barcelone, (Ed) Dopaso, C. D., 553-556, 2000.

Hopfinger, E. J. and Toly, J.-A.: Spatially decaying turbulence and its relation to mixing across density interfaces, J. Fluid Mech., 78, 155-175, 1976.

Kit, E., Strang, E. J., and Fernando, H. J. S.: Measurement of turbulence near shear-free density interfaces, J. Fluid Mech., 334, 293-314, 1997.

Kostinski, A. and Shaw, R. A.: Scale-dependent droplet clustering in turbulent clouds, J. Fluid Mech., 434, 389-398, 2001.

Raffel, M., Willert, C., and Kompenhans, J.: Particle image Velocimetry, Springer, 1998.

Shy, S. S., Tang, C. Y., and Fann, S. Y.: A nearly isotropic turbulence generated by a pair of vibrating grids, Experimental Thermal and Fluid Science, 14, 251-262, 1997.

Srdic, A., Fernando, H. J. S., and Montenegro, L.: Generation of nearly isotropic turbulence using two oscillating grids, Exp. Fluids, 20, 395-397, 1996.

Thompson, S. M. and Turner, J. S.: Mixing across an interface due to turbulence generated by an oscillating grid, J. Fluid Mech., 67, 349-368, 1975.

Yoon, K. and Warhaft, Z.: The evolution of grid-generated turbulence under conditions of stable thermal stratification, J. Fluid Mech., 215, 601-38, 1990. 